\title{
Occult Breast Carcinoma Presenting With Anti-Ri-Associated Paraneoplastic Cerebellar Degeneration Revealed With FDG-PET
}

\author{
Aziz Bazine ${ }^{1}$, Mohamed Fetohi ${ }^{1}$, Maha Ait Berri ${ }^{2}$ Yacir Oufroukhi ${ }^{3}$, Mohamed Ichou $^{4} \&$ Hassan Errihani ${ }^{5}$ \\ ${ }^{1}$ Department of Medical Oncology, Military Hospital My Ismail of Meknès, Morocco \\ ${ }^{2}$ Department of Neurology, Military Hospital Med V of Rabat, Morocco \\ ${ }^{3}$ Center of Radio-Isotopic Explorations Ibn Khaldoun of Rabat, Morocco \\ ${ }^{4}$ Department of Medical Oncology, Military Hospital Med V of Rabat, Morocco \\ ${ }^{5}$ Department of Medical Oncology, National Institute of Oncology of Rabat, Morocco \\ Correspondence: Aziz Bazine; Department of Medical Oncology, Military Hospital My Ismail of Meknès, \\ Meknès, Morocco. Tel: 212-638-900-742. E-mail: bazineaziz@gmail.com
}

Received: August 29, 2014 Accepted: September 29, 2014 Online Published: November 26, 2014

doi:10.5539/cco.v4n1p9 URL: http://dx.doi.org/10.5539/cco.v4n1p9

\begin{abstract}
Paraneoplastic neurological syndromes are rare diseases affecting less than $0,01 \%$ of the cancerous patients. Here, we present a rare case of paraneoplastic cerebellar degeneration with anti-Ri antibodies as the presenting manifestation of an occult breast cancer. Our observation emphasizes the importance of the nature of the anti-neuronal antibody and the fluorodeoxyglucose-positron emission tomography to make a definitive diagnosis in patients with subactue onset of neurological deficit suggesting paraneoplastic cerebellar degeneration.
\end{abstract}

Keywords: breast cancer, cerebellar degeneration, paraneoplastic, anti-Ri antibodies, FDG-PET

\section{Introduction}

Paraneoplastic neurological syndromes (PNS) can be defined as remote effects of cancer that are not caused by the primitive tumor and its metastasis, or by ischemia, infection, nutritional and metabolic deficiencies, surgery or other cancer treatments (Honnorat \& Antoine, 2007). PNS are rare diseases affecting less than $0,01 \%$ of the cancerous patients (Darnell \& Posner, 2003). The most important PNS include cerebellar degeneration, limbic encephalitis, encephalomyelitis, peripheral neuropathy, retinopathy and opsoclonus-myoclonus (Honnorat \& Antoine, 2007; Eseonu, Imtiaz, \& Hogben, 2010). We report a 62-year-old patient who presented with a paraneoplastic cerebellar degeneration (PCD) with anti-Ri antibodies as the presenting manifestation of an occult breast cancer.

\section{Case Report}

A 62-year-old postmenopausal woman presented with a three-month history of progressive unsteady gait, dysarthria, and difficulty in performing daily tasks. Her medical history included arterial hypertension. There were no other systemic or neurological symptoms during this period. Cerebellar syndrome was prominent, including disorders of balance, walking and fast voluntary movement execution. The rest of neurological and physical examination was unremarkable.

Satandard laboratory tests, vitamin E and B12, thyroid hormones, folic acid, and tumor markers such as cancer antigen15-3 (CA15-3), $\alpha$-fetoprotein (AFP), $\beta$-human chorionic gonadotropin (BHCG), carcinoembryonic antigen (CEA), cancer antigen 125 (CA125) and cancer antigen19-9 (CA19-9) in serum were normal. Extensive studies including Sjogren's serology, ANA, ANCA, gliadin antibodies, ceruloplasmin, lyme and toxoplasmosis serology, venereal disease research laboratory (VDRL) test and human immunodeficiency virus (HIV) antibodies were all negative. Magnetic resonance imaging (MRI) of the brain and electroencephalogram (EEG) were unremarkable. Cytology, biochemistry, and microbiology studies of the cerebrospinal fluid (CSF) were normal. Since the above investigations did not provide yield a diagnosis, she was further evaluated for possible paraneoplastic aetiology in view of the rapidly progressing symptoms. Serum and CSF were studied for anti-Yo, anti-Hu, anti-Tr, anti-Ma and anti-Ri antibodies, with positive results for anti-Ri antibodies in both samples. This incited a wide search for malignancy, including computed tomography (CT)-chest/abdomen/pelvis, 
mammography, breast ultrasound and MRI-breast, all normal. Whole body positron emission tomography (PET) using $10 \mathrm{mCi}$ of intravenous fluorodeoxyglucose (FDG) showed an abnormal hot spot in the upper outer quadrant of the left breast.

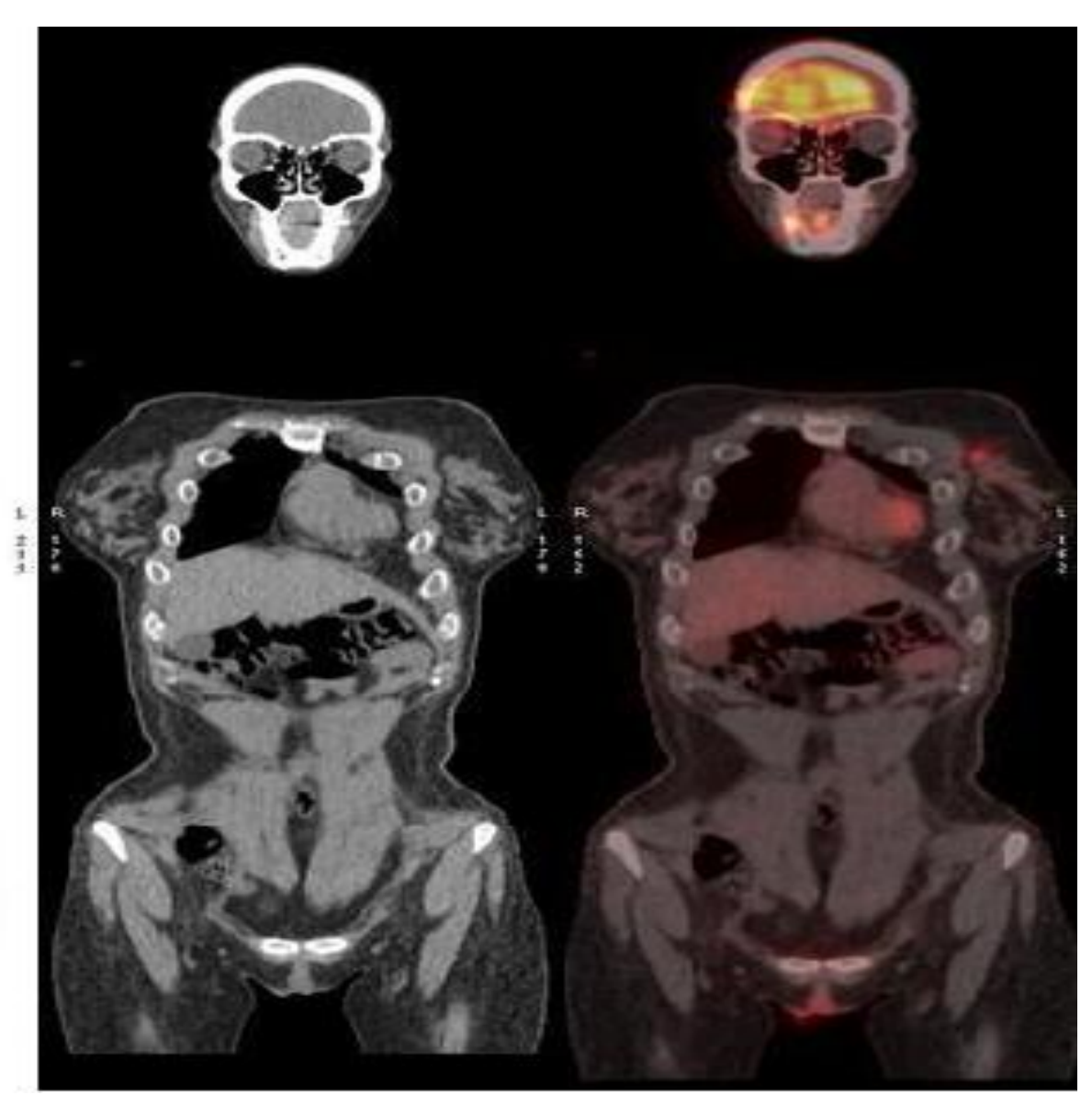

Figure 1. Coronal view of PET, whole body CT and fused images in subject patient showing an abnormal hot spot in the upper outer quadrant of the left breast

The patient initially received intravenous methyl prednisolone ( $1 \mathrm{~g}$ daily for 4 days) with a subjective improvement in her symptoms. After detailed discussion, quadrantectomy and ipsilateral axillary lymphadenectomy were performed. Intraoperative and postoperative pathology confirmed a diagnosis of estrogen and progesterone receptor positive infiltrating ductal carcinoma measuring $1.2 \mathrm{~cm}$ with human epidermal receptor-2 (HER2) overexpression. She subsequently received adjuvant chemotherapy including docetaxel, carboplatin and Herceptin. A neurological follow-up 5 months after initiation of treatment showed a partial clinical improvement of dysarthria and gait. No anti-Ri antibody immunoreactivity was observed in serum or CSF and there was no local or systematic relapse of her neoplastic pathology at that time.

\section{Discussion}

One of the most common and characteristic PNS is PCD (Beukelaar \& Sillevis, 2006). In a study, they analyzed more than 5000 samples for suspicion of PNS, 137 patients had high title antineuronal antibodies and 50 (36\%) of them presented PCD (Brieva-Ruiza et al., 2008). In 1938, the association between PCD and the occult breast or ovary cancers was first identified and Brain described the syndrome completely, for the first time, in 1951 (Brain, Daniel, et Greenfield, 1951; Eseonu et al., 2010; Gatti et al., 2003). There are a few reports of PCD in the literature (Faris, Abraham, \& Barrett-Lee, 1998; Gallegos Sancho et al., 2006; Noorani et al., 2008). On the other hand, $50 \%$ of patients with subacute cerebellar degeneration are estimated to have an underlying malignant disease (Beukelaar \& Sillevis, 2006). The syndrome develops in women over 50 years in nearly two thirds of cases (Gatti et al., 2003). It is characterized by the fast development of grave symptoms including cerebellar ataxia, nystagmus and dysarthria. They appear suddenly, evolve over weeks to months, and then stabilize, often 
leaving the patient significantly impaired and unable to walk or to make the fine movements (Rossato et al., 2013). The symptoms are limited to the cerebellum and its conduction pathway, but other neurological abnormalities can be found on meticulous examination. These include hypoacusia, dysphagia, pyramidal and extrapyramidal signs, peripheral neuropathy, and impaired memory (Beukelaar \& Sillevis, 2006). Cerebellar dysfunction is caused by a significant loss of Purkinje neurons while other cerebellar neurons remain intact. Proteins expressed by tumor cells induce the production of antibodies that have a crossed reaction with similar proteins in central nervous system, leading to a neurological disorder (Rossato et al., 2013; Marchand et al., 2007).

In $60 \%$ of cases, neurological disorders appear before the cancer becomes clinically symptomatic and the patient is referred to a neurologist who has the responsibility to identify these neurological disorders as paraneoplastic (Honnorat \& Antoine, 2007, Marchand et al., 2007). In patients who had a known cancer, it is necessary to eliminate the other causes of cerebellar dysfunction such as metastatsis, nutritional deficits, infection, or side-effects of the anticancer treatment before the diagnosis of PCD is retained (Rutherford, Dineen, et O'Connor, 2007).

Although we observe a cerebellar oedema in some acute cases, the initial brain MRI is usually normal. During this early stage, FDG-PET can show cerebellar hypermetabolism. In the late stage, brain MRI shows cerebellar atrophy while FDG -PET demonstrates hypometabolism (Rutherford et al., 2007, Dalmau \& Rosenfeld, 2008). The diagnosis of PCD is established by detection of specific antineuronal antibodies. The type of antibody directs the search for an underlying tumor (Table 1) (Beukelaar \& Sillevis, 2006).

Table 1. Specific antineuronal antibodies associated with PCD

\begin{tabular}{ll}
\hline Antibody & Associated tumors \\
\hline Anti-Hu (ANNA-1) & SCLC, neuroblastoma, prostate \\
Anti-Yo (PCA-1) & Ovary, breast \\
Anti-CV2 (CRMP5) & SCLC, Thymoma \\
Anti-Ri (ANNA-2) & Breast, SCLC \\
Anti-Ma2 (Ta) & Testicle, lung \\
Anti-Tr (PCA-Tr) & Hodgkin's disease \\
PCA-2 & SCLC \\
Anti-Zic4 & SCLC \\
Anti-mGluR1 & Hodgkin's disease \\
Anti-VGCC & SCLC \\
\hline $\begin{array}{l}\text { antineuronal nuclear antibody; mGluR1 = metabotropic glutamate receptor type 1; PCA = Purkinje } \\
\text { ic antibody; SCLC = small cell lung carcinoma; VGCC = voltage-gated calcium channels. }\end{array}$
\end{tabular}

ANNA = antineuronal nuclear antibody; mGluR1 = metabotropic glutamate receptor type 1; PCA
cytoplasmic antibody; SCLC = small cell lung carcinoma; VGCC = voltage-gated calcium channels.

Anti-Ri is one of the well-characterized onconeural antibody (anti-Hu, Yo, CV2, Ri, MA2) and it is associated with breast cancer in 50\% of cases. Moreover, the diagnosis of PCD should incite the search for an occult cancer independently of the antineuronal antibodies status (Brieva-Ruiza et al., 2008). The identification of anti- Ri antibodies in the serum or CSF should prompt the investigation for a breast or gynecologic cancer, which are screened for by mammogram, followed by bilateral breast MRI for breast cancer and transvaginal ultrasound, followed by $\mathrm{CT}$ of pelvis and abdomen for gynecologic cancer. If no tumors are identified by conventional imaging, as in our case report, FDG-PET is recommended (Brieva-Ruiza et al., 2008; Marchand et al., 2007; Rutherfordet al., 2007; Titulaer et al., 2011). If initial screening is negative in patients presenting PNS with antineuronal antibodies, screening should be repeated after 3-6 months and then every 6 months for 4 years (Titulaer et al., 2011).

There is no standard of care for PCD. Treatment of underlying cancer can lead to improvement of symptoms or at least to stabilize them (Beukelaar \& Sillevis, 2006; Dalmau \& Rosenfeld, 2008). Incidental benefit has been 
reported in association with intravenous immunoglobulin, plasmapheresis, corticosteroid, cyclophosphamide, tacrolimus and rituximab (Eseonu et al., 2010; Beukelaar \& Sillevis, 2006; Dalmau \& Rosenfeld, 2008]. The outcome of PCD is generally poor (Eseonu et al., 2010; Beukelaar \& Sillevis, 2006). Survival after diagnosis is lower in patients with anti-Yo (median 13 months) or anti-Hu (median 7 months) than in patients with anti-Tr (median> 113 months) or anti-Ri (median> 69 months) and the prognosis is better for breast cancers (median 100 months) than for gynecologic cancers (median 22 months) (Eseonu et al., 2010; Beukelaar \& Sillevis, 2006; Dalmau \& Rosenfeld, 2008).

\section{Conclusion}

In summary, we reported a rare case of PCD with anti-Ri onconeural antibody. FDG-PET played an important role in the detection of the occult breast cancer and the neurological symptoms were slightly improved after the surgery and the adjuvant chemotherapy. This case illustrates the challenges concerning the diagnosis of PCD and the relevance of the FDG-PET for identification of the underlying occult cancer.

\section{Disclosure Statement}

The authors have no conflicts of interest to declare.

\section{References}

Beukelaar, J., \& Sillevis, P. (2006). Managing Paraneoplastic Neurological Disorders. The Oncologist, 11, 292-305. http://dx.doi.org/10.1634/theoncologist.11-3-292

Brain, W. R., Daniel, P. M., \& Greenfield, J. G. (1951). Subacute cortical cerebellar degeneration and its relation to carcinoma. Journal of neurology, neurosurgery, and psychiatry, 14(2), 59.

Brieva-Ruiza, L., Diaz-Hurtadoe, M., Matias-Guiub, X., Márquez-Medinac, D., Tarragonab, J., \& Grausd, F. (2008). Anti-Ri-associated paraneoplastic cerebellar degeneration and breast cancer: An autopsy case study. Clinical Neurology and Neurosurgery, 110, 1044-1046. http://dx.doi.org/10.1016/j.clineuro.2008.06.016

Dalmau, J., \& Rosenfeld, M. R. (2008). Paraneoplastic syndromes of the CNS. The Lancet Neurology, 7(4), 327-340. http://dx.doi.org/10.1016/S1474-4422(08)70060-7

Darnell, R. B., \& Posner, J. B. (2003). Paraneoplastic syndromes involving the nervous system. N Engl J Med, 349, 1543-1454. http://dx.doi.org/10.1056/NEJMra023009

Eseonu, K., Imtiaz, F., \& Hogben, K. (2010). A rare case of Breast Carcinoma presenting with Paraneoplastic Cerebellar Degeneration. Journal of Surgical Case Reports, 2010(10), 6-6. http://dx.doi.org/10.1093/jscr/2010.10.6

Faris, M., Abraham, J., \& Barrett-Lee, P. (1998). A patient with breast cancer and paraneoplastic cerebellar syndrome associated with anti-Purkinje cell antibodies: response to CMF chemotherapy. Clinical Oncology, 10(3), 202-203. http://dx.doi.org/10.1016/S0936-6555(98)80072-1

Gallegos Sancho, M. I., López, M. R., Martinez, L. C., Campelo, R. G., López Facal, M. S., Budiño, B. S., ... Antón Aparicio, L. M. (2006). Subacute cerebellar degeneration as paraneoplastic syndrome: initial symptom of breast cancer with HER2 overexpression. Clinical breast cancer, 7(1), 79-80.

Gatti, G., Simsek, S., Kurne, A., Zurrida, S., Naninato, P., Veronesi, P., ... Luini, A. (2003) Paraneoplastic neurological disorders in breast cancer. The Breast, 12, 203-207. http://dx.doi.org/10.1016/S0960-9776(03)00011-0

Honnorat, J., \& Antoine, J. C. (2007). Paraneoplastic neurological syndromes. Orphanet J Rare Dis, 2(1), 22. http://dx.doi.org/10.1186/1750-1172-2-22.

Marchand, V., Graveleau, J., Lanctin-Garcia, C., Bourbouloux, E., Bridji, B., Resche, I. F., ... Rousseau, C. (2007). A rare gynecological case of paraneoplastic cerebellar degeneration discovered by FDG-PET. Gynecologic Oncology, 105, 545-547. 10.1016/j.ygyno.2007.01.047

Noorani, A., Sadiq, Z., Minakaran, N., Coleman, C., Thomas, V. A., \& Mokbel, K. (2008). Paraneoplastic cerebellar degeneration as a presentation of breast cancer-a case report and review of the literature. International Seminars in Surgical Oncology, 5(1), http://dx.doi.org/8. 10.1186/1477-7800-5-8

Rossato, M., Zabeo, E., Burei, M., Cecchin, D., Guzzardo, V., Fassina, A., \& Vettor, R. (2013). Lung Cancer and Paraneoplastic Neurologic Syndromes. Case Report and Review of the Literature. Clinical Lung Cancer, 14(3), 301-9. http://dx.doi.org/10.1016/j.cllc.2012.11.008

Rutherford, G. C., Dineen, R. A., \& O'Connor, A. (2007). Imaging in the investigation of paraneoplastic 
syndromes. Clinical radiology, 62(11), 1021-1035. http://dx.doi.org/10.1016/j.crad.2007.06.005

Titulaer, M. J., Soffietti, R., Dalmau, J., Gilhus, N. E., Giometto, B., Graus, F., ... Verschuuren, J. J. G. M. (2011). Screening for tumours in paraneoplastic syndromes: report of an EFNS task force. European Journal of Neurology, 18(1), 19-e3. http://dx.doi.org/10.1111/j.1468-1331.2010.03220.x

\section{Copyrights}

Copyright for this article is retained by the author(s), with first publication rights granted to the journal.

This is an open-access article distributed under the terms and conditions of the Creative Commons Attribution license (http://creativecommons.org/licenses/by/3.0/). 